La question de la qualité s'invite dans pratiquement tous les domaines de la santé. C'est tout à fait pertinent et nécessaire mais il faut aussi des standards concrets que chacun comprenne et qui puissent être contrôlés. Une simple déclaration du bout des lèvres ne suffit plus aujourd'hui pour convaincre les patients, les fournisseurs de prestations, les assureurs et les milieux politiques. Celui qui ne prend pas son avenir en main se met à la merci des autres.

Dr Remo Osterwalder

Vice-président de la FMH, responsable du département Prestations et développement professionnel

\title{
La qualité dans le sauvetage - un défi complexe
}

\author{
Wolfgang Ummenhofer ${ }^{a}$, Luciano Anselmi ${ }^{b}$, Thomas S. Müller ${ }^{c}$, Gianmaria Solari ${ }^{\text {, }}$ Patrick Siebenpfund ${ }^{e}$, \\ Paul-Martin Sutter ${ }^{f}$
}

a Prof., médecin adjoint Anesthésie, Hôpital universitaire de Bâle, président du Forum du sauvetage de la FMH; b Dr, médecin-chef Anesthésie, Hôpital régional de Bellinzone, président de la Commission qualité de I'IAS; ${ }^{c} \mathrm{Dr}$, médecin-chef du service central des urgences, Hôpital cantonal des Grisons, Coire, membre du Swiss Trauma Board; d Dr, Lugano, représentant de I'IAS au Forum du sauvetage de la FMH; e Dr, Bâle, représentant de la SSMI au Forum du sauvetage de la FMH; ${ }^{\dagger}$ Dr, médecin adjoint Traumatologie, Bienne, directeur national de I'ATLS Switzerland, représentant de la SSC au Forum du sauvetage de la FMH

Les «Thèses de la FMH pour le domaine du sauvetage en Suisse" publiées en 2010 dans le Bulletin des médecins suisses par le Forum du sauvetage de la FMH accordent une place importante à la gestion de la qualité. Le présent article approfondit et précise les différents aspects énoncés au point 8 de ces thèses.

S'agissant de la qualité structurelle du sauvetage, les éléments suivants sont déterminants: respect du délai d'intervention exigé, infrastructure et équipement des services de sauvetage impliqués, répartition professionnalisée des interventions par une centrale d'appels sanitaires d'urgence (CASU), formation du personnel intervenant, choix des secours adaptés et des équipes disponibles pour l'intervention, accompagnement des ambulanciers par un médecin d'urgence si l'indication l'exige, détermination et sélection des hôpitaux cibles appropriés et contrôle qualité des standards fixés.

- Délai d'intervention d'une structure de sauvetage professionnelle: en Suisse, les patients avec un diagnostic vital engagé doivent recevoir des secours professionnels dans un délai de 15 minutes, réduit, si possible, à 10 minutes dans les agglomérations, ce qui est une option réaliste. Cet objectif doit constituer la règle et être respecté dans neuf cas sur dix. Lorsqu'un médecin d'urgence est appelé à intervenir (en fonction de l'indication), il est soumis au même délai. Malgré une forte urbanisation, la Suisse comprend des régions géographiques complexes dans lesquelles ces délais seront difficiles voire impossibles à respecter. Dans ces régions périphériques, un dispositif de garde peut venir compléter le réseau des médecins d'urgence à l'instar de celui mis en place dans les Grisons avec le soutien

\section{Sur le fil:}

- Les patients en Suisse ayant besoin de soins urgents sont pris en charge dans un délai de 15 minutes par une équipe de secours professionnelle; dans les agglomérations, ce délai peut être réduit en général à dix minutes.

- En fonction de l'indication, les ambulanciers formés sont soutenus par des médecins d'urgence au bénéfice d'une AFC en médecine d'urgence préhospitalière (médecin d'urgence SSMUS).

- Dans chaque service de sauvetage en Suisse, la responsabilité médicale incombe au directeur médical qui est au bénéfice $d^{\prime}$ 'une AFC en médecine d'urgence préhospitalière (médecin d'urgence SSMUS).

- Les victimes avec un tableau clinique ou un type de blessure particulier sont orientées directement vers un hôpital cible adapté à leur situation. C'est ce qui a été décidé au niveau politique dans le cadre de la médecine hautement spécialisée pour les patients victimes d'un AVC, de brûlures, de polytraumatisme ou deTCC graves.

- L'IAS est l'instance suisse pour les directives et les certifications des services de sauvetage et des centrales d'appels sanitaires d'urgence (CASU).

- La formation postgraduée en médecine d'urgence est réglée par la SSMUS. En dehors des institutions de formation postgraduée, les patients ayant besoin de soins urgents ne peuvent être pris en charge que par des médecins d'urgence au bénéfice d'une AFC en règle.

- Une gestion judicieuse de la qualité exige le contrôle des interventions de sauvetage sur la base de données pertinentes. Des banques de données anonymisées sont en train de voir le jour pour des groupes de patients spécifiques. Ces registres doivent être institutionnalisés et les données relevées pour l'ensemble du pays.

du canton [1]. Les systèmes de sauvetage au niveau local doivent être reliés aux structures de sauvetage interrégionales. Grâce à l'efficacité des secours aériens de notre pays, il est possible d'offrir des 
délais de prise en charge adaptés aux groupes de patients concernés.

- Directeur médical du service de sauvetage: chaque service de sauvetage en Suisse est dirigé par un médecin d'urgence titulaire d'une attestation de formation complémentaire (AFC) en médecine d'urgence préhospitalière (médecin d'urgence SSMUS). Cette tâche dirigeante ne doit pas se réduire à une position «alibi». Le directeur médical donne des instructions au personnel sous ses ordres pour tout ce qui touche à la médecine. Il se charge de répartir les compétences médicales au personnel non médical et supervise l'assurance qualité du service de sauvetage dans son ensemble.

\section{En Suisse, les patients avec un diagnostic vital engagé doivent recevoir des secours profession- nels dans un délai de 15 minutes.}

- Hôpital cible: après les premiers soins adéquats sur place, les victimes doivent être directement transportées, sous surveillance et traitement, dans un hôpital adéquat pour la prise en charge définitive des atteintes les plus graves. Les cantons ont signé une convention sur la médecine hautement spécialisée (MHS) qui définit explicitement les centres accueillant en urgence les victimes d'AVC, les grands brûlés et les blessés graves; un concept unique approuvé par tous les cantons remplace 26 planifications cantonales différentes. Sa mise en œuvre relève d'un organe scientifique MHS composé de médecins et d'un organe de décision MHS au plan politique. S'agissant des blessés graves, douze centres hospitaliers suisses ont été identifiés pour traiter les patients avec un score ISS (Injury Severity Score) d'au moins 20 points et/ou un traumatisme crânio-cérébral (TCC) grave. La décision MHS impose à ces douze centres, réunis au sein du «Swiss Trauma Board» (STB) en tant que société simple, d'exploiter ensemble le Registre des traumatismes Suisse et de s'engager pour le développement d'un réseau suprarégional des traumatismes. Le degré de sévérité exigé au niveau politique pour qu'un cas soit pris en charge dans un centre des traumatismes MHS constitue encore une entrave au triage préclinique. En effet, l'ISS est un score validé et largement diffusé dans la littérature spécialisée en traumatologie mais c'est une valeur qui se base sur des résultats anatomiques (p. ex. coupes transversales, résultats d'opération) et ne s'applique pas aux soins précliniques. Le mécanisme de l'accident ou les scores physiologiques sont moins précis pour le triage et le patient risque d'être attribué à un degré de priorité respectivement trop faible ou trop élevé. Indépendamment de ça, les patients avec un risque élevé de mortalité (donc aussi les patients urgents dont le diagnostic vital est manifestement engagé) bénéficient d'une prise en charge préhospitalière optimale et de la gestion structurée et bien rodée du centre cible. Ce qui a été démontré pour une importante cohorte du syndrome coronaire aigu [2].

- Directives et recommandations: sur mandat des directeurs cantonaux de la santé, l'Interassociation du sauvetage (IAS) édicte des directives pour les moyens de transport, les équipements des services de sauvetage et le personnel de secours en ce qui concerne l'urgence et la menace vitale des victimes; un ambulancier diplômé et un médecin urgentiste sont présents en fonction de l'indication. Plusieurs dispositions à l'intention des exploitants de centrales d'appels sanitaires d'urgence 144 doivent définir les conditions cadres en Suisse en matière de logistique, à savoir de l'enregistrement à la répartition des interventions. Dans le respect des directives sur la réanimation de 2015, les proches de victimes d'un arrêt cardio-circulatoire hors clinique présumé doivent obtenir des instructions structurées au moment de leur appel au 144 afin de pouvoir exécuter une première réanimation mécanique sur la victime [3].

Chaque service de sauvetage en Suisse est dirigé par un médecin d'urgence titulaire d'une attestation de formation complémentaire (AFC) en médecine d'urgence préhospitalière (médecin d'urgence SSMUS).

- Contrôle de qualité, certifications: il est prévu que tous les services de sauvetage en Suisse soient certifiés IAS ou puissent justifier d'un contrôle de qualité équivalent. La procédure de reconnaissance est lancée par le service de sauvetage ou la CASU qui remplit une auto-déclaration et auquel une équipe d'experts rend visite. Les coûts supplémentaires des services de sauvetage certifiés doivent apparaître dans la structure tarifaire des assureurs; pour autant que les structures reconnues pour leur qualité puissent être au moins en partie reconnues financièrement.

Sur mandat de l'ISFM, la SSMUS est chargée de la formation postgraduée en médecine d'urgence. Elle surveille et procède aux visites des établissements de formation qui délivrent les AFC en médecine d'urgence préhospitalière (médecin d'urgence SSMUS) respectivement des centres d'urgence dans lesquels 
les médecins en formation peuvent accomplir les 50 interventions exigées en tant qu'urgentiste au terme du cursus clinique et après la réussite des cours de préparation (ACLS, PALS, médecin d'urgence). En dehors de ces institutions, les patients ayant besoin de soins urgents ne peuvent être pris en charge que par des médecins d'urgence au bénéfice de l'AFC en médecine d'urgence préhospitalière. Le contrôle qualité des cours de médecin d'urgence et de médecin de garde relève de la SSMUS et respectivement du Forum du sauvetage de la FMH. Le projet «unité de doctrine SFG/CSAM» se penche actuellement sur la réorganisation de la coopération entre le Service sanitaire coordonné (SSC) et la SSMUS pour le cours de «Conduite sanitaire en cas d'événement majeur» (SFG/CSAM) destiné à la formation de médecin-chef des secours (MCS) et d'ambulancier chef des secours (ACS). Les diplômes concernés sont délivrés pour une durée déterminée et doivent faire l'objet d'une recertification après cinq ans. La formation continue obligatoire ou l'activité dans ce domaine est attestée par auto-déclaration et vérifiée de manière ponctuelle. Enfin, l'AFC perd sa validité au bout de six ans si elle n'est pas recertifiée.

- Dispositions relatives à la protection des données: une gestion judicieuse de la qualité exige le contrôle des interventions de sauvetage sur la base de données pertinentes concernant leur indication et adéquation. Actuellement, ces données n'existent pas en Suisse ou elles sont seulement disponibles de manière ponctuelle par région sous la forme de problèmes spécifiques. Jusqu'à présent, leur collecte ne pouvait pas être mise en place pour des raisons compréhensibles liées à la protection des données. La création de registres exige le respect des droits de la personnalité des patients et des dispositions légales en matière de protection des données tout en permettant une saisie anonymisée de caractéristiques définies. Plusieurs institutions, organisations et sociétés de discipline ont commencé à mettre sur pied des banques de données. Comme l'a suggéré l'European Resuscitation Council (ERC), elles devraient recueillir les caractéristiques des diagnostics d'urgence pour ce qu'on appelle communément en anglais le «First Hour Quintet»: (1) ACR, (2) syndrome coronaire aigu, (3) AVC, (4) détresse respiratoire aiguë, (5) polytraumatisme ou TCC grave. Le regard vers l'étranger nous montre qu'il est diffi-

\section{Il est prévu que tous les services de sauvetage en Suisse soient certifiés IAS ou puissent justifier d'un contrôle de qualité équivalent.}

cile de saisir et documenter les caractéristiques des interventions préhospitalières mais aussi que seul un nombre restreint de cas cliniques bien documentés permet d'obtenir une description comparative de la qualité des valeurs correspondant à l'intervention sur place ou au trajet vers l'hôpital. Si un registre coûteux et chronophage doit accueillir des données hors cliniques valides, alors ce ne sera possible dans notre système fédéral du sauvetage très hétérogène que si toutes les institutions, tous les services de secours, CASU, hôpitaux et instances politiques impliqués plaident de manière constructive pour une version uniforme.

\section{Références}

1 Scharplatz D. Der Arzt im Rettungskonzept der Kantone. Schweizerische Ärztezeitung. 2002;83:513-4.

2 Babic D et al. Mortalitätsursachen und Outcome beim akuten Koronarsyndrom. Kardiovaskuläre Medizin. 2015;18:347-54.

32015 American Heart Association Guidelines Update for Cardiopulmonary Resuscitation and Emergency Cardiovascular Care. Circulation. 2015;132, Suppl. 2:pp. 397-413. 